\title{
The Mitochondria-Targeted Metabolic Tubular Injury in Diabetic Kidney Disease
}

\author{
Hong Jiang ${ }^{a, b, c, d, e} \quad X u e S^{a, b, c, d, e} \quad$ Sha Jia ${ }^{a, b, c, c, d, e} \quad$ Lihui Qu $u^{a, b, c, c, d, e}$ \\ Chunhua Weng ${ }^{a, b}, c_{,}, d, e \quad$ Xiujin Shen ${ }^{a, b, c, d, e}$ Yucheng Wang ${ }^{a, b, c, d, e}$ \\ Hongfeng Huang ${ }^{a, b, c, d, e}$ Yingying Wang ${ }^{a, b, b, c, d e} \quad$ Cuili Wang ${ }^{a, b, b, d, e} \quad$ Shi Feng ${ }^{a, b, c, d, e}$ \\ Meizhen Wang ${ }^{f}$ Huajun Feng ${ }^{f}$ Sudarshanee Geekiyanage ${ }^{g}$ \\ Alan James Davidson ${ }^{\mathrm{h}}$ Jianghua Chen ${ }^{\mathrm{a}, \mathrm{b}, \mathrm{c}, \mathrm{d}, \mathrm{e}, \mathrm{e}}$ \\ aKidney Disease Center, the First Affiliated Hospital, College of Medicine, Zhejiang University, \\ Hangzhou, China, 'Key Laboratory of Nephropathy, Hangzhou, China, 'Kidney Disease Immunology \\ Laboratory, the Third-Grade Laboratory, State Administration of Traditional Chinese Medicine of \\ China, Hangzhou, China, ${ }^{~}$ Key Laboratory of Multiple Organ Transplantation, Ministry of Health of \\ China, Hangzhou, China, elnstitute of Nephropathy, Zhejiang University, Zhejiang, China, 'School \\ of Environmental Science and Engineering, Zhejiang Gongshang University, Hangzhou, China, \\ 9Department of Agricultural Biology, Faculty of Agriculture, University of Ruhuna, Mapalana, \\ Kamburupitiya, Sri Lanka, hDepartment of Molecular Medicine \& Pathology, School of Medical \\ Sciences, The University of Auckland, Auckland, New Zealand
}

\section{Key Words}

Diabetic kidney disease (DKD) • Tubular injury $\bullet$ Mitochondria $\bullet$ Metabolomics

\begin{abstract}
Background/Aims: Diabetic kidney disease (DKD) is a leading cause of end-stage renal disease (ESRD) worldwide, and the importance of tubular injury has been highlighted in recent years. However, the underlying mechanisms and effective therapeutic targets are still unclear. In this study, we investigated mtDNA, mitochondrial dynamics, function and metabolic pathways to determine if mitochondrial damage plays a critical role in the development of tubular injury in DKD patients. Methods: A cross-sectional study was carried out among healthy controls $(H C s, n=65)$, diabetes patients without kidney disease $(D C s, n=48)$ and DKD patients $(n=60)$. Serum, peripheral blood mononuclear cells (PBMCs) and kidney biopsy specimens were obtained from participants. Metabolomics was employed to investigate cellular metabolism. Results: DKD patients had decreased mtDNA copy numbers and increased mtDNA damage compared to DCs. Mitochondrial fragmentation was specifically presented in tubules, but not in podocytes of DKD patients. The accumulation of damaged mtDNA and fragmented mitochondria resulted in increased reactive oxygen species (ROS) generation, activation of apoptosis and loss of mitochondrial membrane potential $\left(\Delta \Psi_{\mathrm{m}}\right)$ in tubules and PBMCs. Furthermore, glycolysis and tricarboxylic acid (TCA) cycle was perturbed,

$H$. Jiang and $X$. Shao contributed equally to this work Jiang-Hua Chen Kidney Disease Center, the First Affiliated Hospital, College of Medicine, Zhejiang University and Hong Jiang Qingchun Road 79, Hangzhou 310003 (China)


and increased dihydroxyacetone phosphate (DHAP) and decreased succinyl-CoA synthetase (SCS) respectively in these two metabolic pathways were identified as potential biomarkers for tubular injury in DKD. Conclusion: Our study indicates that mitochondrial damage could be the hallmark of tubular injury in DKD patients, and this would provide a novel and attractive therapeutic target to improve this disease.

\section{Introduction}

Diabetic kidney disease (DKD) represents the largest single cause of end-stage renal disease (ESRD) in Western societies [1,2]. However, the prevalence of DKD has been rapidly increased in recent decades in China, which has become more common than chronic kidney disease (CKD) related to glomerulonephritis [1,3]. This phenomenon has caused profound socioeconomic and public health consequences worldwide [3, 4].

Excessive accumulation of extracellular matrix in glomerular, thickening of tubulointerstitial compartments and hyalinization of intrarenal vasculature are the specific structure changes of DKD [5-7]. Among these features, tubular injury is regarded as part and parcel of pathologic lesion of DKD progression, not only secondary to the changes in the glomerular compartment. In fact, some studies have reported that the extent of tubular injury in DKD, also known as diabetic tubulopathy (dNP), correlates closely with declining kidney function [5-10]. The pathogenetic mechanisms of dNP have been investigated in some literatures, including tubular hypoxia, inhibition of autophagy and generation of reactive oxygen species (ROS). These mechanisms underline the importance of tubular injury in DKD development, which is believed to attract more researchers to explore other novel pathogenetic mechanisms in coming years [5, 9, 11-14].

Mitochondria are the fundamental subcellular organelles in the cytosol of eukaryotic cells that maintain metabolic homeostasis by providing ATP production through oxidative phosphorylation (OXPHOS) [6, 15-18]. The central role of damaged mitochondria in pathogenetic mechanisms of some diseases, such as neurodegenerative, neoplastic, endocrine, and cardiovascular diseases, has been proven by many lines of evidence [17-20]. Kidneys are energetically demanding organs and only second to the heart in mitochondrial abundance. Because of its active reabsorption of metabolites and protein synthesis, proximal tubules use the majority of oxygen for ATP generation and thus contain most of the mitochondria in kidneys [19]. Therefore, mitochondrial dysfunction, bioenergetic defects and dynamic imbalance are believed to play a critical role in tubular injury in DKD development, which has been confirmed by some investigations of patients and animal models $[6,9,15,16,21]$. Meanwhile, other novel mechanisms related to mitochondrial damage, such as metabolic pathways, are still need to be further explored to understand the pathogenesis of DKD more completely.

Metabolomics is a systematic evaluation of small molecules and allows exploration of the nexus of gene-environment interactions in disease pathways. This technology is now frequently used for identifying biomarkers and for elucidating underlying mechanisms in diabetes mellitus (DM) and its complications, including DKD [22-25]. Metabolomic analyses in clinical studies and animal models have suggested that alterations in TCA cycle, fatty acid oxidation and amino acid metabolism are the major pathways affected in DKD. And mitochondrial dysfunction is clearly involved in the changes of these metabolic pathways $[22,26]$. Therefore, our investigation would employ metabolomic analysis to discover some mitochondrial metabolites as unique biomarkers and attractive targets to speculate potential and novel pathogenetic mechanisms of tubular injury in DKD.

In this study, we described the events of mitochondrial dysfunction and dynamic imbalance in the progress of tubular injury in DKD. Furthermore, we explored the alteration of metabolic pathways through metabolomics study and verified the discovered biomarkers to elucidate the prospective pathogenetic mechanisms. These data would provide novel 


\section{Cellular Physiology Cell Physiol Biochem 2019;52:156-171 \\ \begin{tabular}{ll|l} 
and Biol: 10.33594/000000011 & @ 2019 The Author(s). Published by \\
Cell Physiol Biochem Press GmbH\&Co. KG
\end{tabular} \\ Jiang et al.: Mitochondrial Damage in Tubules of DKD}

insights into the fundamental causes and enable the generation of new therapeutic targets of this disease.

\section{Materials and Methods}

\section{Human Subjects}

Patients were enrolled between 2012 and 2016 with written informed consent from the First Affiliated Hospital, College of Medicine, Zhejiang University under ethical approval from the hospital's Institutional Review Committee on Human Research. A random plasma glucose level of $\geq 11.1$ or a fasting plasma glucose (FPG) level of $\geq 7 \mathrm{mmol} / \mathrm{l}$ was considered to be indicative of diabetes $[27,28]$. The patients study groups consisted exclusively of patients with type 2 diabetes (T2D). T2D were defined as follows: onset after age 30 , treated by diet or oral hypoglycaemic agents and/or insulin [27, 28]. The DKD group $(n=60)$ included the patients with the history of T2D and presence of microalbuminuria (albumin 30-300 mg/day, $\mathrm{n}=10$ ) or biopsy-proven DKD ( $n=50)$ [29]. For diabetes controls without nephropathy (DC, $n=48)$ group, we used patients with T2D, no microalbuminuria (albumin $<30 \mathrm{mg} /$ day) and normal renal function [29]. Healthy control (HC, $n=65)$ group included the people with no history of disease or current medication, and their age and gender were matched with the DKD and DC group. Estimated glomerular filtration rate (eGFR) was evaluated using Chronic Kidney Disease Epidemiology Collaboration (CDK-EPI) equation [30]. The clinical baseline characteristics of subjects included in this study was shown in Table 1.

\section{Measurement of Mitochondrial DNA Copy Number by Quantitative Real-Time PCR}

Total genomic DNA was isolated and purified from all serum samples using the Axyprep Blood Genomic DNA Mini Kit (Axygen, Corning, NY, USA). A quantitative real-time PCR-based method was used for the mitochondrial gene ND1 and the nuclear gene $\beta$-actin to determine relative mtDNA copy number. ND1 primers were as follows: 5'-ACACTAGCAGAGACCAACCG-3' (sense) and 5'-GAAGAATAGGGCGAAGGGGC-3' (antisense). B-actin primers were as follows: 5'-TAAAGCGGCCTTGGAGTGTG -3' (sense) and 5'-GAACACGGCTAAGTGTGCTG-3' (antisense). Absolute values of mtDNA and nDNA were carried out in triplicate in the presence of dilution standards using the $\mathrm{iQ}^{\mathrm{TM}} \mathrm{SYBR}^{\circledR}$ Green Supermix (Cat. 1708880, BioRad). MtDNA copy numbers were assessed as ND1/ $\beta$-actin ratio.

\section{Measurement of Mitochondrial DNA Damage}

DNA damage was quantified using the elongase method [31] by comparing the relative amplification of an $8.843 \mathrm{~kb}$ region relative to a $222 \mathrm{bp}$ region in the mitochondrial genome with specific primers. The primers for long PCR were as follows: 5'-TCTAAGCCTCCTTATTCGAGCCGA-3' (sense)

Table 1. Baseline characteristics of healthy control (HC), and diabetic control (DC) and diabetic kidney disease (DKD) group. Data are presented as means \pm SEM and as median (interquartile ranges) for nonnormally distributed data. ${ }^{*} \mathrm{P}<0.05$ compared with $\mathrm{DC} .{ }^{* *} \mathrm{P}<0.01$ compared with $\mathrm{DC} .{ }^{* *} \mathrm{P}<0.001$ compared with DC. BMI, body mass index $\left(\mathrm{kg} / \mathrm{m}^{2}\right)$; FBS, fasting blood sugar ( $\left.\mathrm{mmol} / \mathrm{l}\right)$; ACR, albumin/ creatinine ratio (g/mol); RBP, retinol-binding protein; Cr, creatinine; NR, not recorded; ND, not done

\begin{tabular}{|c|c|c|c|}
\hline Variable & $\begin{array}{l}\text { Healthy control group } \\
\qquad(\mathrm{n}=65)\end{array}$ & $\begin{array}{l}\text { Diabetic control group } \\
\qquad(n=48)\end{array}$ & $\begin{array}{l}\text { Diabetic kidney disease group } \\
\qquad(n=60)\end{array}$ \\
\hline Age (years) & $49.72 \pm 1.52$ & $53.98 \pm 2.32$ & $53.35 \pm 1.51$ \\
\hline Gender(male:female) & $35: 30$ & $29: 19$ & $42: 18$ \\
\hline Diabetes duration (years) & NR & $5.00(9.38)$ & $8.00(7.75)^{*}$ \\
\hline BMI $\left(\mathrm{kg} / \mathrm{m}^{2}\right)$ & ND & $23.43 \pm 0.52$ & $24.21 \pm 0.43$ \\
\hline FBS (mmol/l) & ND & $7.12(3.04)$ & $6.95(5.56)$ \\
\hline $\begin{array}{l}\text { Systolic blood pressure (mm } \\
\mathrm{Hg})\end{array}$ & ND & $123.65 \pm 2.24$ & $149.17 \pm 2.61^{* * *}$ \\
\hline $\begin{array}{l}\text { Diastolic blood pressure }(\mathrm{mm} \\
\mathrm{Hg})\end{array}$ & ND & $75.54 \pm 1.54$ & $86.68 \pm 1.40^{* * *}$ \\
\hline Triglyceride (mmol/l) & ND & $1.12(1.22)$ & $1.68(1.20)^{* *}$ \\
\hline Cholesterol (mmol/l) & ND & $4.12 \pm 0.14$ & $4.91 \pm 0.17^{* *}$ \\
\hline ACR $(\mathrm{g} / \mathrm{mol})$ & ND & $1.63(1.41)$ & $278.21(430.45)^{* * *}$ \\
\hline eGFR (ml min-1 $\left.1.73 \mathrm{~m}^{-2}\right)$ & ND & $100.83 \pm 2.40$ & $60.76 \pm 3.95^{* * *}$ \\
\hline $\mathrm{RBP} / \mathrm{Cr}(\mathrm{g} / \mathrm{mol})$ & ND & $0.02(0.01)$ & $6.09(8.64)^{* * *}$ \\
\hline mtDNA copy number & $11.03(6.84)$ & $11.83(14.37)$ & $9.59(7.31)^{*}$ \\
\hline
\end{tabular}




\section{Cellular Physiology Cell Physiol Biochem 2019;52:156-171 \\ \begin{tabular}{ll|ll} 
and Biochemistry & $\begin{array}{l}\text { DOl: 10.33594/000000011 } \\
\text { Published online: } 28 \text { February } 2019\end{array}$ & $\begin{array}{l}\text { C 2019 The Author(s). Published by } \\
\text { Cell Physiol Biochem Press GmbH\&Co. KG }\end{array}$ \\
\cline { 2 - 3 }
\end{tabular} \\ Jiang et al.: Mitochondrial Damage in Tubules of DKD}

and 5'-TTTCATCATGCGGAGATGTTGGATGG-3' (antisense). The primers for short PCR were as follows: 5'-CCCCACAAACCCCATTACTAAACC-3' (sense) and 5'-TTTCATCATGCGGAGATGTTGGATGG-3' (antisense). The relative amplification of the PCR products were quantified by using Quant-iT PicoGreen dsDNA assay kits (Cat. P11496, Life Technologies) [31] and calculated by normalizing the fluorescence values of the long PCR product to the short PCR product. The decrease in the amplification ratio represented an increase in DNA damage.

Measurement of Apoptosis, ROS Production and Mitochondrial Membrane Voltage Potential ( $\Delta \Psi m)$ by Flow Cytometry

Peripheral blood mononuclear cells (PBMCs) were separated from the blood by density gradient centrifuging over Histopaque 1077 (Cat. 10771, Sigma-Aldrich), and then suspended in PBS at a final concentration of $\sim 10^{5}$ cells/ml for flow cytometry.

Annexin V/propidium iodide (PI) (Cat. 556547, BD Biosciences) was used to measure PBMCs apoptosis. Annexin $\mathrm{V}^{+} / \mathrm{PI}^{-} \mathrm{PBMCs}$ were in early apoptosis and Annexin $\mathrm{V}^{+} / \mathrm{PI}^{+}$in late apoptosis. Cellular ROS production of PBMCs was determined with $\mathrm{H}_{2}$-DCFDA (2', 7'- dichlorodihydrofluorescein diacetate) staining (Cat. C6827, Life Technologies), and evaluated by flow cytometry at 495/530 nm. The dye JC-1 (5, 5' ,6, 6' -Tetrachloro-1, 1' , 3, 3' -tetraethyl-imidacarbocyanine iodide) (Cat. 551302, BD Biosciences) was used for $\Delta \Psi \mathrm{m}$ assessment. JC-1 was able to form J-aggregates within healthy mitochondria with red fluorescence (emission, $590 \mathrm{~nm}$ ) at polarized $\Delta \Psi \mathrm{m}$. In cells with altered mitochondrial function, JC-1 could only form monomers with green fluorescence (emission, $527 \mathrm{~nm}$ ) in cytoplasm at depolarized $\Delta \Psi \mathrm{m}$. The changes of $\Delta \Psi \mathrm{m}$ were recorded by flow cytometer for the determination of cells with green fluorescence. All the stainings were performed following the manufacturer's instructions and analyzed by BD FACS Diva software (BD Biosciences, Franklin Lakes, NJ).

\section{Immunohistochemistry and TUNEL Assay}

Unstained slides of human kidney biopsy tissues were obtained from patients diagnosed as DKD $(n=14)$ and pre-transplant donors as healthy controls $(n=15)$. Unstained sections were processed for immunohistochemical staining with standard protocols. Briefly, after dewaxed and rehydrated, the $1.5-\mu \mathrm{m}$ paraffin-embedded sections were incubated with various primary antibodies: anti-Dynamin-related protein (Drp)-1 (Cat. 611113, BD Biosciences), anti-NADPH oxidase (Nox)-4 (Cat. ab133303, Abcam), anti-Mitofusin (Mfn)-2 (Cat. ab56889, Abcam), anti-Nitrotyrosine polyclonal antibody (Cat. 06-284, EMD Millipore), antiCytochrome c (Cat. 4280, Cell Signaling), anti-Fission (Fis)-1(Cat. sc-98900, Santa Cruz Biotechnology) and anti-Bax (Cat. sc-493, Santa Cruz Biotechnology) antibody. And then secondary antibodies conjugated with peroxidase (GK500705, Gene Co., Shanghai, China). Sections were treated with diaminobenzidine followed by counterstaining with hematoxylin and examined. Five random fields of each section were photographed and the staining was semi-quantified using National Institutes of Health Image J by an investigator blinded to the experimental protocol.

To assess the extent of apoptosis, TUNEL staining was performed using an In Situ Cell Death Detection Kit (Cat. 11684817910, Roche Applied Science), following the manufacturer's instructions. Five random fields of each section were photographed and the mean numbers of apoptotic cells were determined.

\section{Measurement of Mitochondrial Morphology by Electron Microscopy}

Renal cortical and medullary tissues from pre-transplant donors $(n=3)$ and DKD $(n=3)$ group were minced into $1-\mathrm{mm}^{3}$ pieces and processed for electron microscopy with standard protocols. Ultrathin sections (80-90 nm) were prepared for examined and photographed using an Olympus transmission electron microscope (Tecnai, Tokyo, Japan). To quantify the dysmorphic mitochondria, at least 100 mitochondria in proximal tubular epithelial cells from each section were initially identified by an investigator blinded to the experimental protocol. Dysmorphic mitochondria were defined as mitochondria with a focal loss of cristae and fragmented $(<1 \mu \mathrm{m}$ in length) $[6,32]$.

\section{Serum Metabolomic Analysis}

The serum samples were mixed with the extraction liquid $\left(350 \mu \mathrm{L}\right.$, methanol/acetonitrile $/ \mathrm{dH}_{2} \mathrm{O}, 1 / 2 / 2$, $\mathrm{v} / \mathrm{v} / \mathrm{v})$ and an internal standard $\left(20 \mu \mathrm{L} \mathrm{L}\right.$-2-Chlorophenylalanineas, $1 \mathrm{mg} / \mathrm{ml}$ stock in $\left.\mathrm{dH}_{2} \mathrm{O}\right)$. After dried and resuspended, the supernatant was prepared for analysis by liquid chromatography (LC) mass spectrometry 


\section{Cellular Physiology Cell Physiol Biochem 2019;52:156-171 \\ \begin{tabular}{ll|ll} 
and Biochemistry & $\begin{array}{l}\text { DOl: 10.33594/000000011 } \\
\text { Published online: } 28 \text { February } 2019\end{array}$ & $\begin{array}{l}\text { O } 2019 \text { The Author(s). Published by } \\
\text { Cell Physiol Biochem Press GmbH\&Co. KG }\end{array}$ \\
\cline { 2 - 3 }
\end{tabular} \\ Jiang et al.: Mitochondrial Damage in Tubules of DKD}

(MS) in Q Exactive Orbitrap (Thermo Fisher Scientific, USA). Metabolites were identified and quantified based on in-house database using Tracefinder v3.1 (Thermo Fisher Scientific, USA), and statistical analyses of metabolites were further performed using MetaboAnalyst (http://www.metaboanalyst.ca). Briefly, fold change analysis in means of metabolites between the studied groups was conducted. The significance of the differences in metabolites between the groups was determined in a multivariate linear-regression model (volcano plot) after the metabolites were transformed -10 logarithms by Student's t-tests. BenjaminiHochberg step-down approach was used for false discovery rate correction during multiple comparisons. Metabolites with significantly different levels among the groups $(p<0.05)$ were selected to conduct metabolite set enrichment analysis (MSEA) by KEGG (Kyoto Encyclopedia of Genes and Genomes, www. genome.jp/kegg/). The pathways with $p \leq 0.05$ in Bernoulli distribution are defined as the pathways which were significantly enriched by the metabolites with levels significantly changed among the groups.

The levels of oxaloacetate (OAA), dihydroxyacetone phosphate (DHAP) and succinyl-CoA synthetase (SCS) activity were detected using the assay kits (Cat. ab83428, ab197003 and ab196989, Abcam), according to the manufacturer's instructions

\section{Statistical Analyses}

Statistical analyses for the baseline characteristics of participants, mtDNA copy number and damage, flow cytometry, mitochondrial morphology, immunohistochemistry and validation of metabolites were carried out using SPSS software (version 19.0) and GraphPad Prism 5.0 software (GraphPad Inc., San Diego, CA, USA). Normally distributed data were presented as mean \pm SEM, and analyzed using parametric test (2 groups, Student's $t$-test; > 2 groups, one-way ANOVA). Non-normally distributed data were presented as median (interquartile ranges), and analyzed using non-parametric test ( 2 groups, Mann-Whitney test; $>2$ groups, Kruskal Wallis with Dunn- Bonferroni posthoc test). Spearman's tests were applied to determine the correlation of multiple variables with renal tubular injury in DC and DKD patients. $P<0.05$ was considered statistically significant.

\section{Results}

\section{MtDNA damage was increased in serum of DKDs}

Three groups of subjects were compared using a cross-sectional study design: healthy controls (HCs, $n=65$ ) comprised of the people with no history of disease, diabetes controls without kidney disease (DCs, $n=48$ ) included the T2D patients with normal renal function and no history of albuminuria, and diabetic kidney disease patients (DKDs, $n=60$ ) with a current albuminuria (Table 1). Groups were matched for age and gender. The DKD patients had longer duration of diabetes, higher albumin/creatinine ratio (ACR), retinol-binding protein (RBP)/ creatinine ( $\mathrm{Cr}$ ) ratio, systolic and diastolic blood pressure, triglyceride and cholesterol, and lower eGFR than DCs.

We found that DCs had significantly higher mtDNA copy numbers compared to DKD patients $(p=0.036)$, whereas no significant difference between HCs and DKDs (Fig. 1A). It has been suggested that mtDNA comprises of both intact and damaged molecules [15]. Therefore, to confirm the existence of heteroplasmy in mtDNA, a PCR with elongase method was used to quantify the level of damaged mtDNA. Relative amplification of mtDNA was significantly lower in DKD compared to HC $(p<0.0001)$ and DC $(p=0.005)$ (Fig. 1B), which demonstrated that the mtDNA in the serum samples of DKDs mainly consisted of damaged mtDNA. Further analysis revealed a negative correlation of mtDNA copy number $(r=-0.259$ $p=0.007$, Fig. 1C) and amplification of mtDNA ( $\mathrm{r}=-0.375 \mathrm{p}<0.0001$, Fig. 1D) with urinary $\mathrm{RBP} / \mathrm{Cr}$ ratio respectively. RBP has been widely used as the biomarker of tubular injury [33, 34].

\section{Mitochondria were fragmented in proximal tubules of DKDs}

The status of mitochondrial morphology in kidney biopsy tissues was assessed by electron microscopy (EM). In the proximal tubules of DKD group, mitochondria fragmented into short rods or spheres and had cristolysis, compared with the elongated mitochondria 
with organized cristae observed in HC group (Fig. 2A-D). Quantification through morphometric analysis revealed that the percentage of dysmorphic mitochondria was significantly increased in DKD group ( $p=0.017$, Fig. 2E). The expression of mitochondrial-shaping proteins was assessed by immunohis tochemistry. The mitochondrial fission proteins Drp1 and Fis1 were notably up-regulated, and the fusion protein Mfn2 levels were decreased (Fig. 2F-K). These changes in the expression of mitochondrial dynamic proteins showed the excessive fission of mitochondria in the proximal tubules of DKD, which were consistent with the increase of fragmented mitochondria in electron micrographs. There was no significant difference of the mitochondrial morphology in podocytes between $\mathrm{HC}$ and

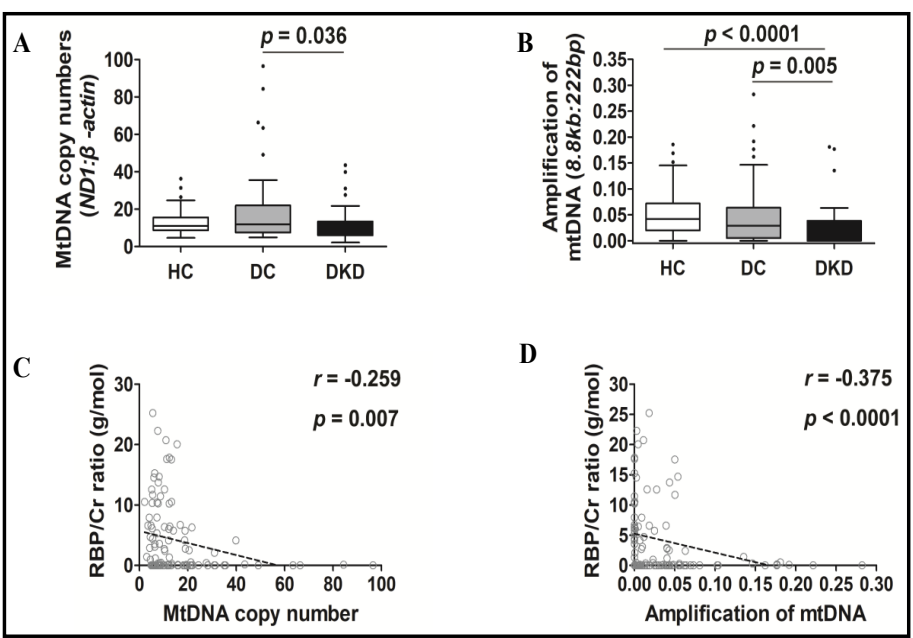

Fig. 1. MtDNA damage was increased in serum of DKDs. DNA were isolated from serum of HCs $(n=65)$, DCs $(n=48)$ and DKDs $(n=60)$. (A) MtDNA copy numbers in DKD patients were significantly decreased compared to DC patients. A real time qPCR was carried out to determine mtDNA copy numbers as mitochondrial (ND1) to nuclear ( $\beta$-actin) ratio. (B) Damaged mtDNA was significantly increased in DKD patients. DNA damage was quantified using the elongase method. The relative amplification was quantified by normalizing the fluorescence values of the long PCR product $(8.843 \mathrm{~kb})$ to the short PCR product (222 bp). (C and D) Correlation analysis revealed mtDNA copy number and amplification of mtDNA was negatively correlated with RBP /Cr ratio, respectively. r, correlation coefficient. RBP, retinol-binding protein; Cr, creatinine; HC, healthy control; DC, diabetic control; DKD, diabetic kidney disease. DKD group (Supplementary

Fig. 1 - all supplementary material available online at www.cellphysiolbiochem.com).

\section{Mitochondria appeared dysfunctional in proximal tubules and PBMCs of DKDs}

Accumulation of mtDNA and disruption of the balance between mitochondrial fission and fusion would inevitably lead to mitochondrial dysfunction, activation of apoptosis and exacerbation of oxidative stress $[17,35]$. TUNEL assay was used to observe the activation of apoptosis, and the expression of apoptogenic proteins was assessed by immunohistochemistry. The percentage of apoptotic cells was significantly increased in proximal tubules of DKD group compared to HC group (Fig. 3A and B). Bax and Cytochrome $\mathrm{C}$ levels, the main proteins in two steps of apoptosis that involve mitochondria [17] were both significantly up-regulated (Fig. 3C-F). Since there was no renal injury in DC patients, we could not acquire their informed consents to get the kidney tissues using renopuncture. Some previous researches have suggested that PBMCs from patients would resemble systemic changes in the body and could be used as surrogate cells for kidney tissue [15]. Therefore, mitochondrial function, ROS production and apoptosis in kidney of DC group could also be evaluated in PBMCs. The flow cytometry analysis of PI/FITC staining demonstrated that the apoptotic PBMCs were significantly increased in DKDs compared to HCs $(p=0.002)$ and DCs ( $p=0.032$ ) (Fig. 3G and H), which were consistent with the increase of apoptotic cells in proximal tubules. Correlation analysis indicated a positive correlation between apoptotic PBMCs and RBP/Cr ratio ( $r=0.472, p=0.007$, Fig. $3 \mathrm{I}$ ).

The protein levels of Nox4 and nitrotyrosine, the oxidation species-generating enzyme and indicator of nitrosative stress respectively, were assessed to observe ROS production in proximal tubules. These two proteins were both significantly up-regulated in DKDs compared 
to HCs (Fig. 4A-D). And the ROS levels in PBMCs, which were marked by H2DCFDA staining, were also significantly increased in DKDs compared to HCs ( $p=$ $0.038)$ and DCs ( $p=0.024)$ (Fig. 4E and F). In addition, mitochondrial dysfunction characterized by a notable loss of mitochondrial voltage potential $(\Delta \Psi \mathrm{m})$ was seen in PBMCs of DKDs compared to HCs ( $p=$ $0.024)$, which was assessed by JC-1 staining (Fig. 4H and I). ROS production ( $r$ $=0.633, p=0.037$, Fig. 4G) and loss of $\Delta \Psi \mathrm{m}$ in PBMCs $(r=0.847, p=0.016$, Fig. 4J) was found positively associated with $\mathrm{RBP} / \mathrm{Cr}$ ratio, respectively.

\section{Glycolysis and} tricarboxylic acid (TCA) cycle was perturbed in serum of $D K D$ s

A total of 1043 separate metabolites were measured in HC $(n=30)$, DC $(\mathrm{n}=27)$ and $\mathrm{DKD}$ group $(n=30)$. The three groups were distributed separately in a principle component analysis (PCA)

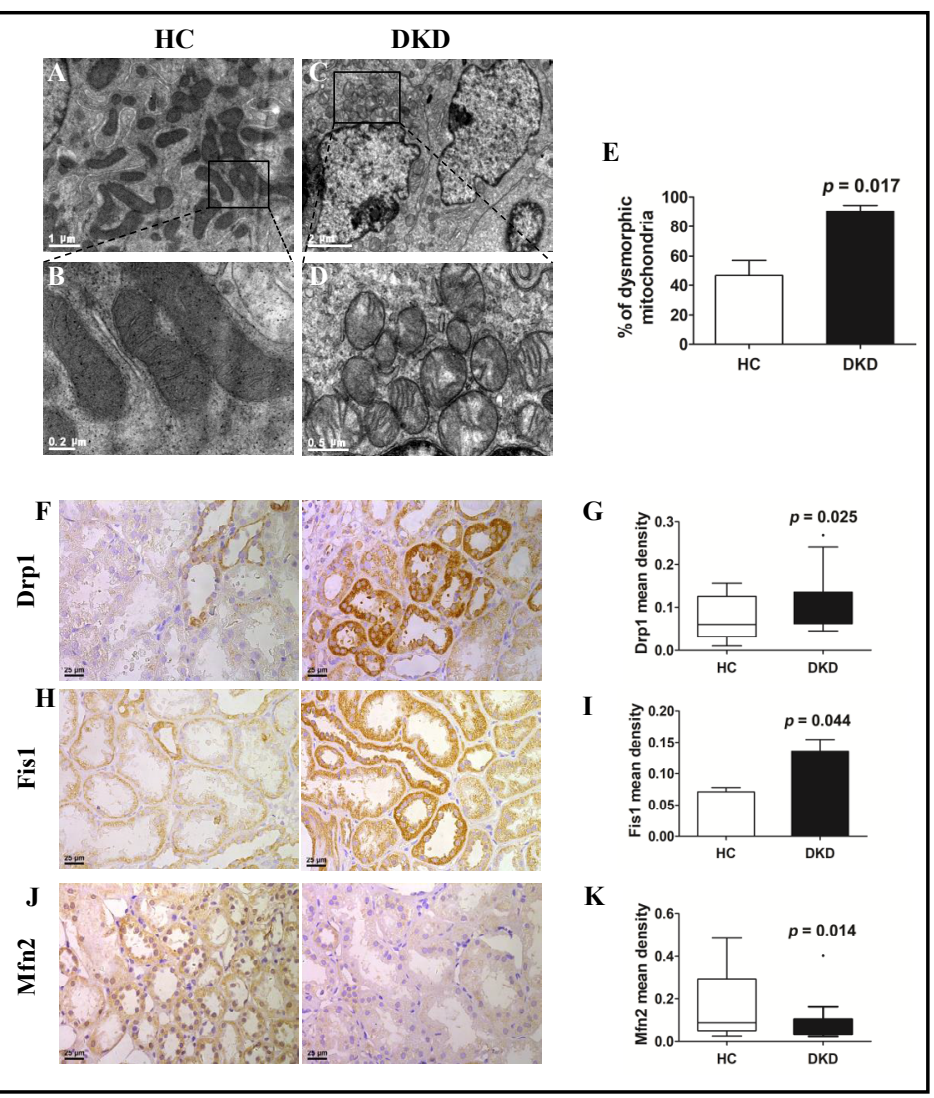

Fig. 2. Mitochondria were fragmented in proximal tubules of human kidney biopsy tissues from DKDs. (A-D) By electron microscopy, mitochondria fragmented into short rods or spheres in DKDs $(n=3)$, and the elongated mitochondria with organized cristae observed in HCs $(n=3)$. (E) The mitochondrial changes in electron micrographs were quantified and included in bar graphs. (F-K) The mitochondrial fission protein Drp1 and Fis1 was notably increased and the fusion protein Mfn2 was decreased in renal tubules compared to HCs. $\times 400$ magnification. HC, healthy control; DKD, diabetic kidney disease. (Supplementary Fig. 2A).

291 metabolites were found significantly different in DKD group compared with DC group (Supplementary Fig. 2B and Supplementary Table 1), and 22 of them were identified to enrichment in mainly 7 metabolism pathways by KEGG analysis (Fig. 5A, Supplementary Fig. 2C and D). By searching KEGG database and literatures, 3 metabolites ultimately, which are the components of glycolysis and tricarboxylic acid (TCA) cycle, were chosen as the mitochondrial metabolism related biomarkers in DKDs: the levels of DHAP and succinyl-CoA were increased and OAA were decreased (Fig. 5B). And the validation experiments of these 3 metabolites were also carried out by the assay kits. The results of the validation experiments were that the levels of DHAP were increased in DKDs compared to HCs ( $p<0.0001$, Fig. 6A), OAA levels were not significantly changed (Fig. 6B), and the activity of SCS was significantly increased in DCs compared to HCs $(p=0.004)$, and decreased in DKDs compared to DCs $(p=0.001$, Fig. 6C). SCS is an enzyme that catalyzes the reversible conversion of succinylCoA to succinate (Fig. 5B) [36], thus the changes of SCS levels are opposite to that of succinylCoA. In conclusion, the alterations of DHAP and succinyl-CoA levels in DKDs were consistent with the results of metabolomics analysis, and OAA levels were not significantly changed. Correlation analysis showed a positive correlation of DHAP $(r=0.237, p=0.014$, Fig. 6D) 


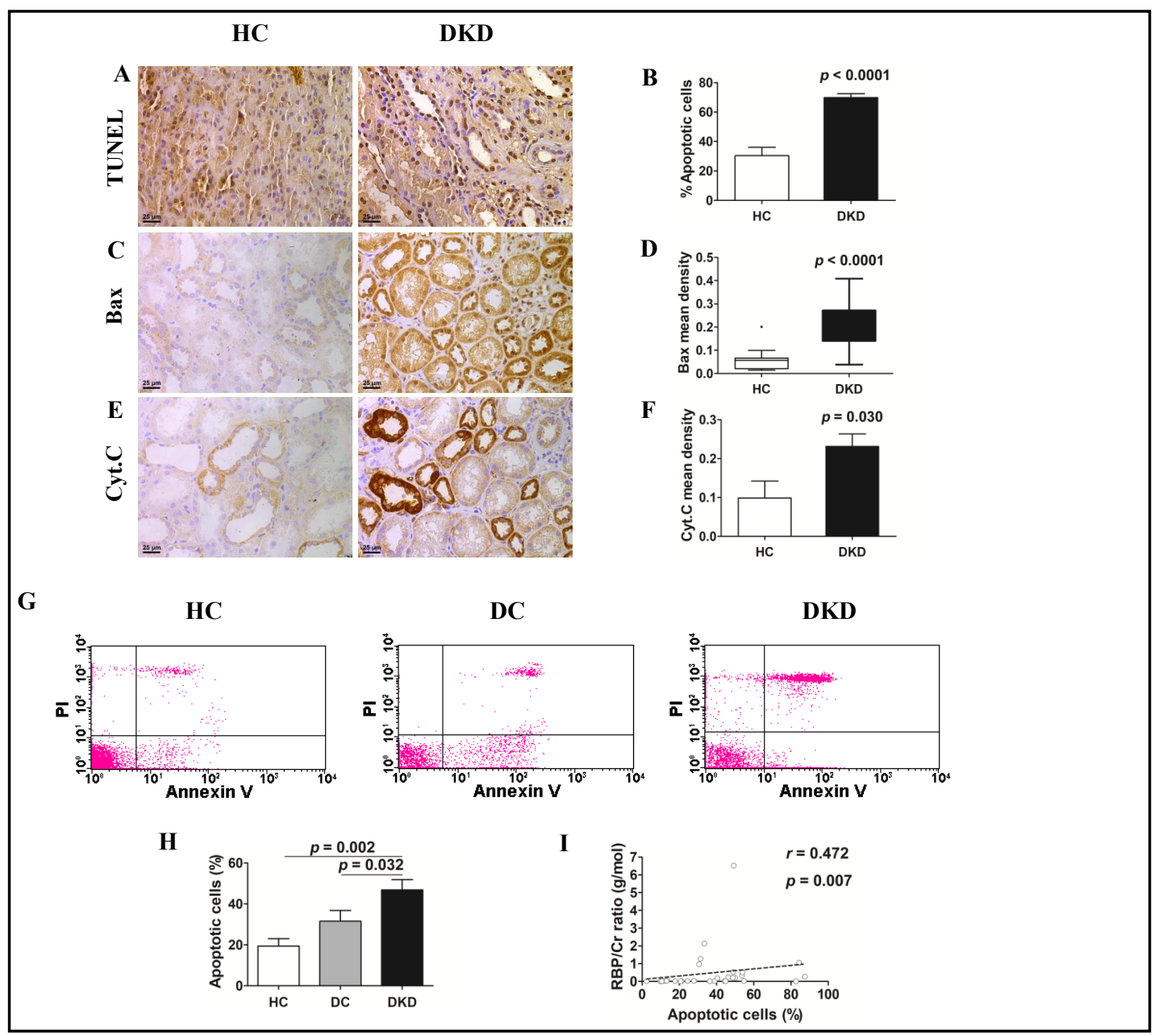

Fig. 3. Apoptosis was activated in proximal tubules and PBMCs of DKDs. (A and B) TUNEL procedure indicated an increase in apoptotic cells in renal tubules in DKDs compared to HCs. $\times 400$ magnification. (CF) The expression levels of Bax and Cytochrome $\mathrm{c}$ were both significantly up-regulated in renal tubules in DKDs compared to HCs. $\times 400$ magnification. (G and H) Flow cytometric analysis showed increased apoptotic PBMCs from DKDs ( $n=14)$ compared to HCs ( $n=9)$ and DCs $(n=19)$. (I) Correlation analysis revealed apoptotic PBMCs was positively correlated with RBP /Cr ratio. r, correlation coefficient; RBP, retinol-binding protein; Cr, creatinine; HC, healthy control; DC, diabetic control; DKD, diabetic kidney disease; Cyt.C, Cytochrome c.

and OAA levels ( $r=0.281, p=0.003$, Fig. $6 \mathrm{E}$ ) with $\mathrm{RBP} / \mathrm{Cr}$ ratio respectively, and a negative correlation between SCS levels and RBP $/ \mathrm{Cr}$ ratio ( $r=-0.298, p=0.002$, Fig. $6 \mathrm{~F}$ ).

\section{Discussion}

This study has suggested that mitochondrial damage contribute to the development of tubular injury in DKD patients. Accumulation of damaged mtDNA, together with excessive mitochondrial fission that regulated this process, would give rise to mitochondrial dysfunction and ROS overproduction within cells, and apoptosis and disruption of cellular metabolism at the whole-cell level.

Human mitochondrial DNA (mtDNA) is a circular molecule of 16, 569 bp that encodes essential protein subunits of oxidative phosphorylation system, which drives mitochondrial respiration and provides ATP production [37]. Due to close proximity to the free radicals producing electron transport chain and lack of nucleotide excision repair, mtDNA is highly 


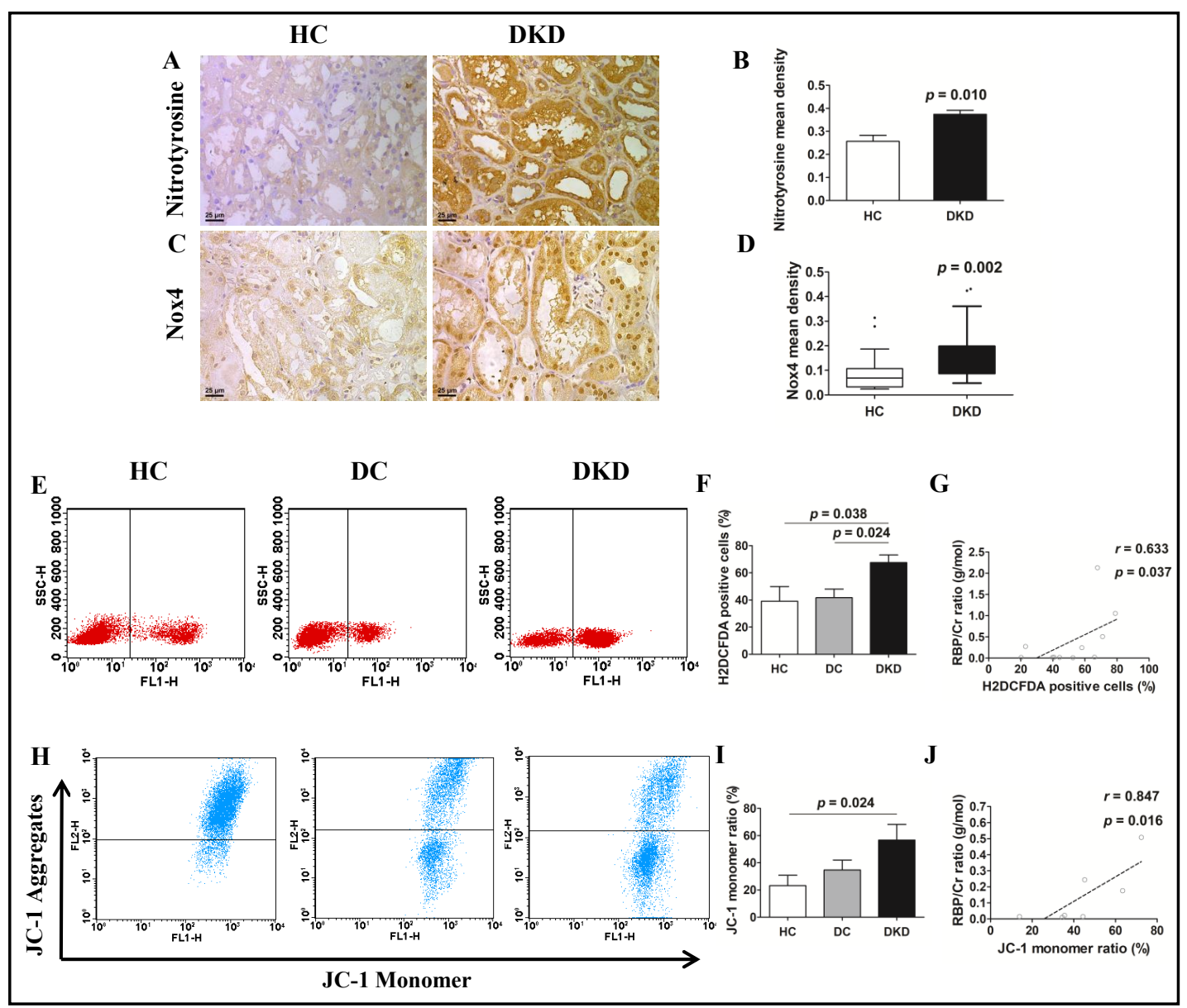

Fig. 4. ROS production was enhanced and mitochondrial membrane potential was decreased in proximal tubules and PBMCs of DKDs. (A-D) Expression of nitrotyrosine and Nox4 were both significantly upregulated in renal tubules in DKDs compared to HCs. $\times 400$ magnification. (E, F, H and I) Flow cytometric analysis showed overproduction of ROS and loss of mitochondrial membrane potential $(\Delta \Psi \mathrm{m})$ in PBMCs from DKDs (ROS, $n=4 ; \Delta \Psi \mathrm{m}, \mathrm{n}=3$ ) compared to HCs (ROS, $\mathrm{n}=3 ; \Delta \Psi \mathrm{m}, \mathrm{n}=5$ ) and DCs (ROS, $\mathrm{n}=7 ; \Delta \Psi \mathrm{m}, \mathrm{n}=4$ ). (G and J) Correlation analysis revealed ROS production and loss of $\Delta \Psi \mathrm{m}$ was positively correlated with RBP /Cr ratio, respectively. r, correlation coefficient; RBP, retinol-binding protein; $\mathrm{Cr}$, creatinine; $\mathrm{HC}$, healthy control; DC, diabetic control; DKD, diabetic kidney disease.

susceptible to ROS induced damage and mutations $[37,38]$. The central role of mtDNA damage and mutations in pathogenetic mechanisms of common human diseases, such as age-related disease and inflammation, has been proven [37, 39]. MtDNA damage and mutations have been considered as a potential cause of diabetes [40]. Hyperglycemia-induced excessive oxidative stress in diabetes patients [41] leads to the changes in mitochondrial biogenesis, which contribute to altered mtDNA abundance [42], and DNA itself becomes damaged resulting in accumulation of mutations. In current study, serum samples were obtained to further investigate the changes of cell-free circulating mtDNA copy numbers and mtDNA damage in diabetes patients [43]. We found that DKD patients had lower mtDNA copy numbers than DCs, with increased mtDNA damage (Fig. 1A and B). We speculated that low levels of oxidative stress caused by hyperglycaemia in DCs could be an adaptive response that increased mtDNA copy numbers to compensate for mitochondria dysfunction and to sustain oxidative phosphorylation. Long term and high levels of oxidative stress in DKDs might eventually decrease mtDNA copy numbers alongside the increased damaged mtDNA $[17,37,42,44]$. 


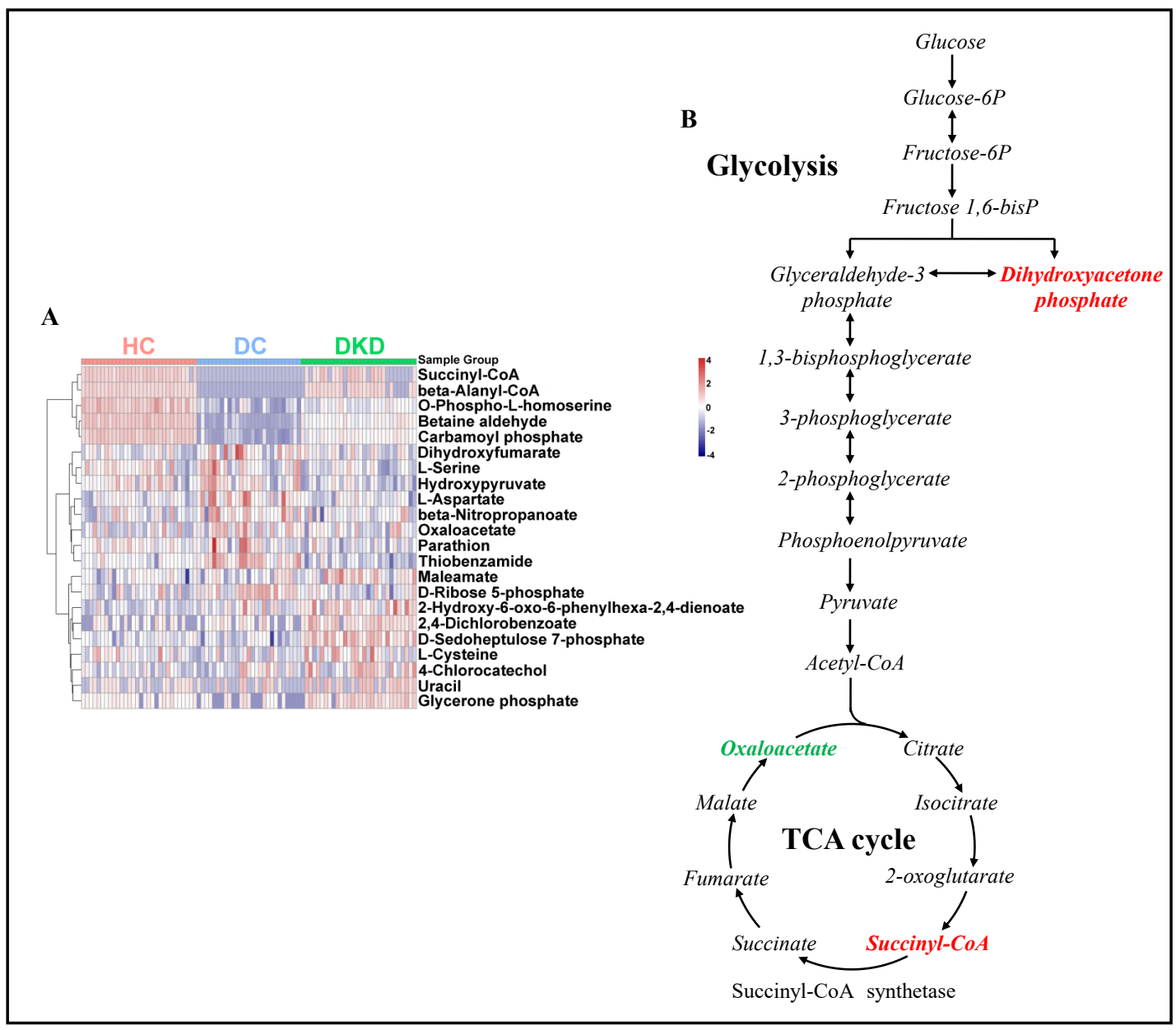

Fig. 5. Glycolysis and tricarboxylic acid (TCA) cycle was perturbed in serum of DKDs. (A) Heatmap (red=higher, green=lower) of 22 significantly different metabolites in HCs $(n=30), D C s(n=27)$ and DKDs $(n=30)$. (B) Schematic of glycolysis and TCA cycle involving 3 metabolites. Red denote increased levels and green denote decreased levels. HC, healthy control; DC, diabetic control; DKD, diabetic kidney disease; TCA cycle, tricarboxylic acid cycle.

MtDNA copy number, integrity and distribution is maintained and regulated by mitochondrial dynamic processes [45]. Mitochondria are highly dynamic organelles that continuously undergo fusion and fission, which make up an integrated quality control axis with mitophagy to maintain mitochondrial homeostasis in response to stress [6, 15-18]. In a number of studies, inhibition of mitochondrial dynamics, especially fusion deficiencies, results in accumulation of damaged mtDNA, impaired mitochondrial function, and eventually cell death $[35,45]$. In this study, we found that most of the mitochondria within tubules in DKD patients were fragmented (Fig. 2A-E), accompanied with up-regulated fission proteins and down-regulated fusion protein (Fig. $2 \mathrm{~F}-\mathrm{K}$ ), which suggested the imbalance between the rates of mitochondrial fission and fusion. However, mitochondrial morphology in podocytes of DKD patients was not significantly changed compared with HCs (Supplementary Fig. 1). This result might be controversial with previous reports of diabetic mouse models [46]. Thus, we supposed that disruption of mitochondrial dynamics might have no significant effect on podocyte injury, but be specific for tubular injury in DKD patients. In addition, mtDNA copy number has been suggested as a biomarker of abnormal mitochondrial dynamics and dysfunction $[42,45]$, and mitochondrial damage plays a critical role in tubular injury in DKD $[6,21]$. Therefore, we suggested that decreased mtDNA copy number and increased mtDNA damage might be regarded as the potential biomarkers of tubular injury in DKD patients. 


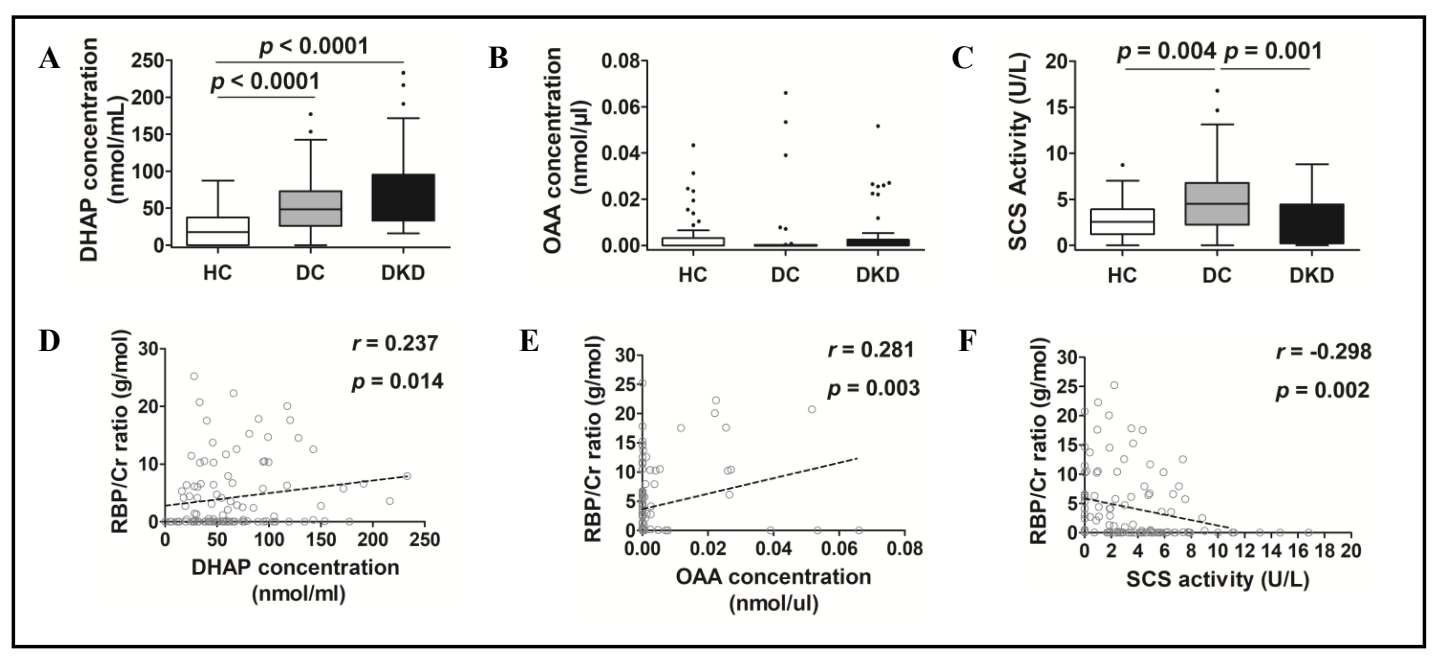

Fig. 6. Validation experiments of DHAP, OAA and SCS levels in serum of DKDs. (A) DHAP levels were increased in DKDs ( $n=60)$ compared to HCs $(n=65)$, (B) OAA levels were not significantly changed, and (C) the activity of SCS was significantly increased in DCs $(n=48)$ compared to HCs $(n=65)$, and decreased in DKDs $(n=60)$ compared to DCs. (D-F) Correlation analysis of 3 metabolites with tubular injury. DHAP (D) and OAA levels (E) were positively correlated with tubular injury respectively, SCS levels (F) were negatively correlated with tubular injury. r, correlation coefficient. RBP, retinol-binding protein; $\mathrm{Cr}$, creatinine; HC, healthy control; DC, diabetic control; DKD, diabetic kidney disease; SCS, succinyl-CoA synthetase; DHAP, dihydroxyacetone phosphate; OAA, oxaloacetate.

To verify this hypothesis, we investigate the association between mtDNA and tubular injury by correlation analyses. A negative correlation between mtDNA copy number and amplification of mtDNA and tubular injury was found (Fig. 1C and D). Hence, in future study, we would further explore the specificity of mtDNA as a biomarker and predictor of tubular injury in DKD. According to the theories proposed before that circulating cell-free mtDNA is increasingly regarded as a mitochondrial damage-associated molecular pattern (DAMP) which would stimulate a cellular anti-inflammatory response and cause organ injury [37, 42, 47], it would be interesting to investigate the mechanisms involved in the release of mtDNA from the injured tubular cells to circulation in DKD.

An increasing body of evidence have suggested that progressive accumulation of damaged mtDNA and abnormal mitochondrial dynamics (usually excessive fission or/and deficient fusion) would trigger ROS overproduction, which, in turn, deteriorate mitochondrial health. Eventually, this self-perpetuating vicious cycle would be account for evitable apoptotic cell death at the whole-cell level $[17,40,48]$. In our study, accompanied with mtDNA damage and excessive mitochondrial fission, the expression of mitochondrial function-related proteins Nox4, Bax and Cytochrome c was significantly increased in the tubules (Fig. 3A-F and 4A-D). It has been proven that the NADPH oxidases of the Nox family are a major source of ROS in diabetic kidney $[12,41]$. As a member of the Nox family, Nox4 is unique as it is primarily localized in the mitochondria and the most abundant Nox isoform in the renal system, and its activity would result in the overproduction of mitochondrial ROS (mtROS) [12, 49, 50] (Fig. $4 \mathrm{C}$ and $\mathrm{D}$ ). Then the accumulation of mtROS stimulated the translocation of proapoptotic protein Bax and the release of the essential component of the respiratory chain Cytochrome c from mitochondria (Fig. 3C-F), which triggered the mitochondria-mediated apoptosis pathways $[51,52]$ and led to the increase in apoptotic tubular cells in DKD patients (Fig. $3 \mathrm{~A}$ and $\mathrm{B}$ ). Moreover, mtROS overproduction and activation of mitochondria-mediated apoptosis would induce mitochondrial dysfunction [35, 53], which would form a vicious cycle $[48,54]$. Because PBMCs can be used as surrogate cells for kidney tissue [15], and alterations of mitochondrial function in PBMCs better correlate with that in kidney tissues than in other organs [55], we also performed detection of mitochondria on them. Consistent 


\section{Cellular Physiology Cell Physiol Biochem 2019;52:156-171 \\ \begin{tabular}{ll|l} 
and Biochemistry $10.33594 / 000000011$ & Published online: 28 February 2019 & 2019 The Author(s). Published by \\
Cell Physiol Biochem Press GmbH\&Co. KG
\end{tabular} \\ Jiang et al.: Mitochondrial Damage in Tubules of DKD}

with the results in tubules, enhanced ROS production (Fig. 4E and F), activation of apoptosis (Fig. 3G and $\mathrm{H}$ ) and concomitant mitochondrial dysfunction characterized by $\Delta \Psi \mathrm{m}$ loss (Fig. $4 \mathrm{H}$ and I) was discovered in DKD group. Additionally, fragmented mitochondria accumulated specifically in tubules (Fig. 1, 2 and Supplementary Fig. 1), and apoptosis, ROS production and loss of $\triangle \Psi \mathrm{m}$ in PBMCs were positively correlated with tubular injury (Fig. 3I, 4G and J). Thus we supposed that mitochondrial damage in PBMCs could reflect that in tubules, and could be regarded as a novel indicator of tubular injury in DKD. Meanwhile, as PBMCs represent a systemic change in the body [15], and the altered mitochondrial function in PBMCs has also been found in other diseases [56,57], therefore, further studies are still needed to determine specific mitochondrial changes in PBMCs associated with tubular injury in DKD, and to explore the molecular mechanism of this change. To sum up, our findings provided the evidence that mitochondrial dysfunction accompanied by the overproduction of mtROS and activation of mitochondria-mediated apoptosis could be the critical mechanism involved in the tubular injury in DKD patients.

Damaged mtDNA and fragmented mitochondria result in perturbed cellular metabolism which may underlie the mitochondrial dysfunction $[39,45,58,59]$. Therefore, a metabolomics analysis was employed to investigate the changes in cellular metabolism in DKD. As mitochondrial damage was the hallmark of tubular injury in DKD patients in our study, we focused on the TCA cycle and glycolysis, which are integrated to catabolize glucose to provide energy for cellular function [60]. We found that DHAP and succinyl-CoA was increased and OAA decreased in serum from DKD patients compared with DC patients (Fig. 5A and B). DHAP is the substrate for isomerization to glyceraldehyde-3-phosphate in glycolysis [60]. The increased generation of DHAP in DKD patients indicated excessive glycolysis which is not a benign sequence of reaction in cancer, aging, Parkinson's disease and other diseases $[58,61]$. It has been suggested that the increase in glycolysis is a compensatory effect of maintaining ATP synthesis $[61,62]$, which was reflected by the increased DHAP accompanied with mitochondrial dysfunction and the disrupted TCA cycle in DKD patients in our study. Succinyl-CoA and OAA are both the metabolic intermediates in the TCA cycle. Conversion of succinyl-CoA to succinate is catalyzed by SCS with simultaneous formation of GTP [60]. According to this fact, we speculated that the increase of SCS activity in DCs in our validation experiments (Fig. 6C) might be a compensatory response for maintaining the normal succinyl-CoA levels. But because of the more severe mitochondrial dysfunction, the SCS activity in DKDs eventually decreased (Fig. 6C), resulting in the accumulation of succinyl-CoA in TCA cycle. It has been reported that the loss of SCS subunit would lead to mitochondrial damage and development of some diseases [63, 64]. Thus, succinyl-CoA accumulation and SCS deficiency might be the important break points in the TCA cycle in DKD patients. Although the decrease in OAA levels was not verified in our validation experiments, a positive correlation of OAA levels with tubular injury was found (Fig. 6E). Furthermore, OAA treatment has been reported effective in neuron damage, stroke and diabetes [65], so the role of OAA in progression of DKD could not yet be negated. Since correlation analysis revealed that DHAP and SCS levels were positively and negatively associated with tubular injury, respectively (Fig. 6D and F), we supposed that DHAP and SCS could be identified as the potential biomarkers for tubular injury in DKD, revealing the specific mitochondrial damage in tubules. As the prominent contribution of specific tissues to changes of metabolites in circulation has been reported $[66,67]$, we would jointly conduct metabolomics analysis of serum, kidney tissue and tubule cells in vivo and in vitro in future research, to explore the specificity of DHAP and SCS as biomarkers of tubular injury in DKD, and the mechanism of changes of metabolites in circulation caused by this injury. 


\section{Conclusion}

In our study, mitochondrial damage was considered as the hallmark of tubular injury in DKD patients. Furthermore, 2 metabolites in glycolysis and TCA cycle were identified as potential biomarkers. This study of mitochondria would provide a novel and attractive therapeutic target to improve tubular injury in DKD.

\section{Acknowledgements}

This work was supported by the grants National Natural Science Foundation of China (81470938, 81770697, 81770752, 81770750), and by the grant Zhejiang Provincial Natural Science Foundation of China (LQ18H050003).

\section{Disclosure Statement}

The authors have no conflicts of interest to disclose.

\section{References}

- 1 Forbes JM, Cooper ME: Mechanisms of diabetic complications. Physiol Rev 2013;93:137-188.

- 2 Xue R, Gui D, Zheng L, Zhai R, Wang F, Wang N: Mechanistic Insight and Management of Diabetic Nephropathy: Recent Progress and Future Perspective. J Diabetes Res 2017;2017:1839809.

3 Zhang L, Long J, Jiang W, Shi Y, He X, Zhou Z, Li Y, Yeung RO, Wang J, Matsushita K, Coresh J, Zhao MH, Wang H: Trends in Chronic Kidney Disease in China. N Engl J Med 2016;375:905-906.

4 Zhang L, Wang F, Wang L, Wang W, Liu B, Liu J, Chen M, He Q Liao Y, Yu X, Chen N, Zhang JE, Hu Z, Liu F, Hong D, Ma L, Liu H, Zhou X, Chen J, Pan L, et al.: Prevalence of chronic kidney disease in China: a crosssectional survey. Lancet 2012;379:815-822.

5 Gilbert RE: Proximal Tubulopathy: Prime Mover and Key Therapeutic Target in Diabetic Kidney Disease. Diabetes 2017;66:791-800.

6 Zhan M, Usman IM, Sun L, Kanwar YS: Disruption of renal tubular mitochondrial quality control by Myoinositol oxygenase in diabetic kidney disease. J Am Soc Nephrol 2015;26:1304-1321.

7 Kanwar YS, Sun L, Xie P, Liu FY, Chen S: A glimpse of various pathogenetic mechanisms of diabetic nephropathy. Annu Rev Pathol 2011;6:395-423.

8 Xiao L, Xu X, Zhang F, Wang M, Xu Y, Tang D, Wang J, Qin Y, Liu Y, Tang C, He L, Greka A, Zhou Z, Liu F, Dong Z, Sun L: The mitochondria-targeted antioxidant MitoQ ameliorated tubular injury mediated by mitophagy in diabetic kidney disease via Nrf2/PINK1. Redox Biol 2017;11:297-311.

9 Sharma I, Dutta RK, Singh NK, Kanwar YS: High Glucose-Induced Hypomethylation Promotes Binding of Sp-1 to Myo-Inositol Oxygenase: Implication in the Pathobiology of Diabetic Tubulopathy. Am J Pathol 2017;187:724-739.

10 Nath KA: Tubulointerstitial changes as a major determinant in the progression of renal damage. Am J Kidney Dis 1992;20:1-17.

11 De Nicola L, Gabbai FB, Liberti ME, Sagliocca A, Conte G, Minutolo R: Sodium/glucose cotransporter 2 inhibitors and prevention of diabetic nephropathy: targeting the renal tubule in diabetes. Am J Kidney Dis 2014;64:16-24.

12 Gorin Y, Block K: Nox4 and diabetic nephropathy: with a friend like this, who needs enemies? Free Radic Biol Med 2013;61:130-142.

13 Ha H, Lee HB: Reactive oxygen species and matrix remodeling in diabetic kidney. J Am Soc Nephrol 2003;14:S246-249.

14 Ding Y, Choi ME: Autophagy in diabetic nephropathy. J Endocrinol 2015;224:R15-30. 


\section{Cellular Physiology Cell Physiol Biochem 2019;52:156-171 \begin{tabular}{ll|ll} 
and Biochemistry & $\begin{array}{l}\text { DOl: 10.33594/000000011 } \\
\text { Published online: } 28 \text { February } 2019\end{array}$ & $\begin{array}{l}\text { C 2019 The Author(s). Published by } \\
\text { Cell Physiol Biochem Press GmbH\&Co. KG }\end{array}$ \\
\cline { 2 - 3 }
\end{tabular} \\ Jiang et al.: Mitochondrial Damage in Tubules of DKD}

- 15 Czajka A, Ajaz S, Gnudi L, Parsade CK, Jones P, Reid F, Malik AN: Altered Mitochondrial Function, Mitochondrial DNA and Reduced Metabolic Flexibility in Patients With Diabetic Nephropathy. EBioMedicine 2015;2:499-512.

- 16 Coughlan MT, Nguyen TV, Penfold SA, Higgins GC, Thallas-Bonke V, Tan SM, Van Bergen NJ, Sourris KC, Harcourt BE, Thorburn DR, Trounce IA, Cooper ME, Forbes JM: Mapping time-course mitochondrial adaptations in the kidney in experimental diabetes. Clin Sci (Lond) 2016;130:711-720.

- 17 Youle RJ, van der Bliek AM: Mitochondrial fission, fusion, and stress. Science 2012;337:1062-1065.

- 18 Archer SL: Mitochondrial dynamics--mitochondrial fission and fusion in human diseases. N Engl J Med 2013;369:2236-2251.

- 19 Forbes JM: Mitochondria-Power Players in Kidney Function? Trends Endocrinol Metab 2016;27:441-442.

- 20 Chinnery PF: Mitochondrial disease in adults: what's old and what's new? EMBO Mol Med 2015;7:15031512.

21 Xiao L, Zhu X, Yang S, Liu F, Zhou Z, Zhan M, Xie P, Zhang D, Li J, Song P, Kanwar YS, Sun L: Rap1 ameliorates renal tubular injury in diabetic nephropathy. Diabetes 2014;63:1366-1380.

22 Sharma K, Karl B, Mathew AV, Gangoiti JA, Wassel CL, Saito R, Pu M, Sharma S, You YH, Wang L, DiamondStanic M, Lindenmeyer MT, Forsblom C, Wu W, Ix JH, Ideker T, Kopp JB, Nigam SK, Cohen CD, Groop PH, et al.: Metabolomics reveals signature of mitochondrial dysfunction in diabetic kidney disease. J Am Soc Nephrol 2013;24:1901-1912.

- 23 Tokarz J, Haid M, Cecil A, Prehn C, Artati A, Moller G, Adamski J: Endocrinology Meets Metabolomics: Achievements, Pitfalls, and Challenges. Trends Endocrinol Metab 2017;28:705-721.

- 24 Freeman OJ, Unwin RD, Dowsey AW, Begley P, Ali S, Hollywood KA, Rustogi N, Petersen RS, Dunn WB, Cooper GJ, Gardiner NJ: Metabolic Dysfunction Is Restricted to the Sciatic Nerve in Experimental Diabetic Neuropathy. Diabetes 2016;65:228-238.

25 Wishart DS: Emerging applications of metabolomics in drug discovery and precision medicine. Nat Rev Drug Discov 2016;15:473-484.

26 Darshi M, Van Espen B, Sharma K: Metabolomics in Diabetic Kidney Disease: Unraveling the Biochemistry of a Silent Killer. Am J Nephrol 2016;44:92-103.

27 Alberti KG, Zimmet PZ: Definition, diagnosis and classification of diabetes mellitus and its complications. Part 1: diagnosis and classification of diabetes mellitus provisional report of a WHO consultation. Diabet Med 1998;15:539-553.

28 WHO: Definition and diagnosis of diabetes mellitus and intermediate hyperglycemia: Report of a WHO/IDF consultation. WHO, 2006, pp 9-12. URL:

https://apps.who.int/iris/bitstream/handle/10665/43588/9241594934_eng.pdf;jsessionid=A1DADBDC4 5A7CD06F2484ECFAC434D6E?sequence=1.

- 29 Stevens PE, Levin A: Evaluation and management of chronic kidney disease: synopsis of the kidney disease: improving global outcomes 2012 clinical practice guideline. Ann Intern Med 2013;158:825-830.

30 Levey AS, Stevens LA, Schmid CH, Zhang YL, Castro AF, 3rd, Feldman HI, Kusek JW, Eggers P, Van Lente F, Greene T, Coresh J: A new equation to estimate glomerular filtration rate. Ann Intern Med 2009;150:604612.

- 31 Furda A, Santos JH, Meyer JN, Van Houten B: Quantitative PCR-based measurement of nuclear and mitochondrial DNA damage and repair in mammalian cells. Methods Mol Biol 2014;1105:419-437.

- 32 Mallipattu SK, Horne SJ, D’Agati V, Narla G, Liu R, Frohman MA, Dickman K, Chen EY, Ma'ayan A, Bialkowska AB, Ghaleb AM, Nandan MO, Jain MK, Daehn I, Chuang PY, Yang VW, He JC: Kruppel-like factor 6 regulates mitochondrial function in the kidney. J Clin Invest 2015;125:1347-1361.

33 Fiseha T, Tamir Z: Urinary Markers of Tubular Injury in Early Diabetic Nephropathy. Int J Nephrol 2016;2016:4647685.

34 Norden AG, Lapsley M, Unwin RJ: Urine retinol-binding protein 4: a functional biomarker of the proximal renal tubule. Adv Clin Chem 2014;63:85-122.

35 Zhan M, Brooks C, Liu F, Sun L, Dong Z: Mitochondrial dynamics: regulatory mechanisms and emerging role in renal pathophysiology. Kidney Int 2013;83:568-581.

- 36 Matilainen S, Isohanni P, Euro L, Lonnqvist T, Pihko H, Kivela T, Knuutila S, Suomalainen A: Mitochondrial encephalomyopathy and retinoblastoma explained by compound heterozygosity of SUCLA2 point mutation and 13q14 deletion. Eur J Hum Genet 2015;23:325-330. 


\section{Cellular Physiology Cell Physiol Biochem 2019;52:156-171 \begin{tabular}{l|l|l|l|l}
\hline DOI: 10.33594/000000011 2019 The Author(s). Published by &
\end{tabular} and Biochemistry Published online: 28 February 2019 Cell Physiol Biochem Press GmbH\&Co. KG \\ Jiang et al.: Mitochondrial Damage in Tubules of DKD}

- 37 West AP, Shadel GS: Mitochondrial DNA in innate immune responses and inflammatory pathology. Nat Rev Immunol 2017;17:363-375.

38 Gustafsson CM, Falkenberg M, Larsson NG: Maintenance and Expression of Mammalian Mitochondrial DNA. Annu Rev Biochem 2016;85:133-160.

- 39 Stewart JB, Chinnery PF: The dynamics of mitochondrial DNA heteroplasmy: implications for human health and disease. Nat Rev Genet 2015;16:530-542.

- 40 Taylor RW, Turnbull DM: Mitochondrial DNA mutations in human disease. Nat Rev Genet 2005;6:389-402.

- 41 Singh DK, Winocour P, Farrington K: Oxidative stress in early diabetic nephropathy: fueling the fire. Nat Rev Endocrinol 2011;7:176-184.

42 Malik AN, Czajka A: Is mitochondrial DNA content a potential biomarker of mitochondrial dysfunction? Mitochondrion 2013;13:481-492.

43 Thierry AR, El Messaoudi S, Gahan PB, Anker P, Stroun M: Origins, structures, and functions of circulating DNA in oncology. Cancer Metastasis Rev 2016;35:347-376.

44 Mishra M, Lillvis J, Seyoum B, Kowluru RA: Peripheral Blood Mitochondrial DNA Damage as a Potential Noninvasive Biomarker of Diabetic Retinopathy. Invest Ophthalmol Vis Sci 2016;57:4035-4044.

45 Meyer JN, Leuthner TC, Luz AL: Mitochondrial fusion, fission, and mitochondrial toxicity. Toxicology 2017;391:42-53.

- 46 Wang W, Wang Y, Long J, Wang J, Haudek SB, Overbeek P, Chang BH, Schumacker PT, Danesh FR: Mitochondrial fission triggered by hyperglycemia is mediated by ROCK1 activation in podocytes and endothelial cells. Cell Metab 2012;15:186-200.

- 47 Liu J, Zou Y, Tang Y, Xi M, Xie L, Zhang Q, Gong J: Circulating cell-free mitochondrial deoxyribonucleic acid is increased in coronary heart disease patients with diabetes mellitus. J Diabetes Investig 2016;7:109-114.

- 48 Jezek J, Cooper KF, Strich R: Reactive Oxygen Species and Mitochondrial Dynamics: The Yin and Yang of Mitochondrial Dysfunction and Cancer Progression. Antioxidants (Basel) 2018;7:pii:E13.

- 49 Block K, Gorin Y, Abboud HE: Subcellular localization of Nox4 and regulation in diabetes. Proc Natl Acad Sci U S A 2009;106:14385-14390.

50 Belarbi K, Cuvelier E, Destee A, Gressier B, Chartier-Harlin MC: NADPH oxidases in Parkinson's disease: a systematic review. Mol Neurodegener 2017;12:84.

51 Kim R, Emi M, Tanabe K: Role of mitochondria as the gardens of cell death. Cancer Chemother Pharmacol 2006;57:545-553.

52 Polster BM, Fiskum G: Mitochondrial mechanisms of neural cell apoptosis. J Neurochem 2004;90:12811289.

- 53 Ikemoto H, Tani E, Ozaki I, Kitagawa H, Arita N: Calphostin C-mediated translocation and integration of Bax into mitochondria induces cytochrome c release before mitochondrial dysfunction. Cell Death Differ 2000;7:511-520.

- 54 Hosseinzadeh A, Kamrava SK, Joghataei MT, Darabi R, Shakeri-Zadeh A, Shahriari M, Reiter RJ, Ghaznavi H, Mehrzadi S: Apoptosis signaling pathways in osteoarthritis and possible protective role of melatonin. J Pineal Res 2016;61:411-425.

- 55 Karamercan MA, Weiss SL, Villarroel JP, Guan Y, Werlin E, Figueredo R, Becker LB, Sims C: Can peripheral blood mononuclear cells be used as a proxy for mitochondrial dysfunction in vital organs during hemorrhagic shock and resuscitation? Shock 2013;40:476-484.

56 Maestraggi Q Lebas B, Clere-Jehl R, Ludes PO, Chamaraux-Tran TN, Schneider F, Diemunsch P, Geny B, Pottecher J: Skeletal Muscle and Lymphocyte Mitochondrial Dysfunctions in Septic Shock Trigger ICUAcquired Weakness and Sepsis-Induced Immunoparalysis. Biomed Res Int 2017;2017:7897325.

57 Dell'Anna ML, Urbanelli S, Mastrofrancesco A, Camera E, Iacovelli P, Leone G, Manini P, D'Ischia M, Picardo M: Alterations of mitochondria in peripheral blood mononuclear cells of vitiligo patients. Pigment Cell Res 2003;16:553-559.

58 Nagdas S, Kashatus DF: The Interplay between Oncogenic Signaling Networks and Mitochondrial Dynamics. Antioxidants (Basel) 2017;6:pii:E33.

- 59 Georgieva E, Ivanova D, Zhelev Z, Bakalova R, Gulubova M, Aoki I: Mitochondrial Dysfunction and Redox Imbalance as a Diagnostic Marker of "Free Radical Diseases". Anticancer Res 2017;37:5373-5381.

60 Bhagavan NV, Ha CE: Chapter 12 - Carbohydrate Metabolism I : Glycolysis and the Tricarboxylic Acid Cycle. Essentials of Medical Biochemistry, ed 2. Academic Press, Elsevier, 2011, pp 115-133. 


\section{Cellular Physiology and Biochemistry}

Cell Physiol Biochem 2019;52:156-171

61 Hipkiss AR: On the Relationship between Energy Metabolism, Proteostasis, Aging and Parkinson's Disease: Possible Causative Role of Methylglyoxal and Alleviative Potential of Carnosine. Aging Dis 2017;8:334-345.

62 Czajka A, Malik AN: Hyperglycemia induced damage to mitochondrial respiration in renal mesangial and tubular cells: Implications for diabetic nephropathy. Redox Biol 2016;10:100-107.

63 Zhao Y, Tian J, Sui S, Yuan X, Chen H, Qu C, Du Y, Guo L, Du H: Loss of succinyl-CoA synthase ADP-forming beta subunit disrupts mtDNA stability and mitochondrial dynamics in neurons. Sci Rep 2017;7:7169.

64 Pedersen BA, Yazdi PG, Taylor JF, Khattab OS, Chen YH, Chen Y, Wang PH: Novel remodeling of the mouse heart mitochondrial proteome in response to acute insulin stimulation. Nutr Metab Cardiovasc Dis 2015;25:1152-1158.

65 Wilkins HM, Swerdlow RH: Relationships Between Mitochondria and Neuroinflammation: Implications for Alzheimer's Disease. Curr Top Med Chem 2016;16:849-857.

66 Giesbertz P, Padberg I, Rein D, Ecker J, Hofle AS, Spanier B, Daniel H: Metabolite profiling in plasma and tissues of ob/ob and db/db mice identifies novel markers of obesity and type 2 diabetes. Diabetologia 2015;58:2133-2143.

67 Varma VR, Oommen AM, Varma S, Casanova R, An Y, Andrews RM, O’Brien R, Pletnikova O, Troncoso JC, Toledo J, Baillie R, Arnold M, Kastenmueller G, Nho K, Doraiswamy PM, Saykin AJ, Kaddurah-Daouk R, Legido-Quigley C, Thambisetty M: Brain and blood metabolite signatures of pathology and progression in Alzheimer disease: A targeted metabolomics study. PLoS Med 2018;15:e1002482. 